\title{
Antioxidant, Anti-inflammatory, and Neuroprotective Effects of Novel Vinyl Sulfonate Compounds as Nrf2 Activator
}

\author{
Ji Won Choi, ${ }^{\dagger, \perp}$ Su Jeong Shin, ${ }^{\dagger, \dagger, \perp}$ Hyeon Ji Kim, ${ }^{\dagger, \dagger}$ Jong-Hyun Park, ${ }^{\dagger}$ Hyeon Jeong Kim, ${ }^{\dagger,}$ \\ Elijah Hwejin Lee, ${ }^{\dagger}$ Ae Nim Pae, ${ }^{\dagger, \S, \|}$ Yong Sun Bahn, ${ }^{\dagger}$ and Ki Duk Park ${ }^{*}, \dagger, \S, \|$ (])
}

${ }^{\dagger}$ Convergence Research Center for Diagnosis, Treatment and Care System of Dementia, Korea Institute of Science and Technology (KIST), Seoul 02792, Republic of Korea

"Department of Biotechnology, Yonsei University, Seoul 03722, Republic of Korea

${ }^{\S}$ Division of Bio-Medical Science \& Technology, KIST School, Korea University of Science and Technology, Seoul 02792, Republic of Korea

"KHU-KIST Department of Converging Science and Technology, Kyung Hee University, Seoul 02447, Republic of Korea

\section{Supporting Information}

ABSTRACT: The main pathway responsible for cellular regulation against oxidative stress is nuclear factor E2-related factor-2 (Nrf2) signaling. We previously synthesized and reported a novel vinyl sulfone (1) as an Nrf2 activator with therapeutic potential for Parkinson's disease (PD). In this study, we changed the vinyl sulfone to vinyl sulfonamide or vinyl sulfonate to improve Nrf2 activating efficacy. We observed that the introduction of vinyl sulfonamide led to a reduction of the effects on Nrf2 activation, whereas vinyl sulfonate compounds exhibited superior activity compared to the vinyl sulfone compounds. Among the vinyl sulfonates, $3 \mathrm{c}$ exhibited 6.9- and 83.5-fold higher effects on Nrf2 activation than the corresponding vinyl sulfone (1) and vinyl sulfonamide (2c), respectively. Compound 3c was confirmed to induce expression of the Nrf2-dependent antioxidant enzymes at the protein level in cells. In addition, 3c mitigated PD-associated behavioral deficits by protecting DAergic neurons in the MPTP-induced mouse model of PD.

KEYWORDS: Parkinson's disease, antioxidant, anti-inflammatory, Nrf2/Keap1 pathway, MPTP mouse model, vinyl sulfone
$\mathrm{P}$ arkinson's disease (PD) is the most prevalent neurodegenerative disorder featured clinically by motor deficits. PD patients have clinical features such as tremor, rigidity, slowness of movement, and postural disability. ${ }^{1,2}$ Most of the symptoms are caused by the death of dopaminergic (DAergic) neurons that produce chemical messengers called dopamine in the substantia nigra ( $\mathrm{SN}$ ) pars compacta. ${ }^{3,4}$ The etiology and pathogenesis of PD is not yet known, but several studies have suggested that oxidative stress is partly responsible for the loss of DAergic neurons and plays an important role in neurodegeneration of PD. ${ }^{5-7}$ Another factor contributing to the pathogenesis of $\mathrm{PD}$ has been thought to be neuroinflammation. Microglial activation is linked to the loss of DAergic neurons, suggesting that neuronal inflammation contribute to the progressive degeneration. ${ }^{8,9}$ Several clinical studies have reported that the levels of inflammatory enzymes have been elevated in mouse models of PD and brain of PD patients. $^{10-12}$

Nuclear factor E2-related factor-2 (Nrf2), encoded by gene NFE2L2, is a nuclear transcription factor that regulates cellular defense system against oxidative stress and has been shown to down-regulate inflammatory responses. ${ }^{13,14}$ Under normal conditions, Nrf2 adheres to Kelch-like ECH-associated protein 1 (Keap1) as a complex and undergoes ubiquitination and proteasomal degradation in cytoplasm. Under oxidative stress conditions, Nrf2 is liberated from Keap1 and translocated into the nucleus, which binds to the antioxidant response element (ARE) sequence in the DNA promoter region and begins transcription of the ARE-regulated antioxidant enzyme such as heme oxygenase-1 (HO-1), glutamate-cysteine ligase (GCL), and reduced nicotinamide adenine dinucleotide phosphate $(\mathrm{NAD}(\mathrm{P}) \mathrm{H})$ quinone oxidoreductase 1 (NQO1). ${ }^{15}$

Although the precise mechanism associated with the Nrf2mediated anti-inflammation pathway has not been elucidated in detail, recent studies reported that inflammatory mediators were overexpressed in the Nrf2 knockout mice and deficiency in transcription factor $\mathrm{Nrf2}$ worsens inflammatory parameters in a mouse model of neurodegenerative diseases. ${ }^{18-20}$ The sole

Received: April 9, 2019

Accepted: June 3, 2019

Published: June 3, 2019 
FDA-approved Nrf2 activator, dimethyl fumarate (DMF, brand name Tecfidera), shows effectiveness in treating multiple sclerosis (MS) by regulating inflammation. ${ }^{21,22}$ Taken together, targeting the Nrf2 pathway is an attractive therapeutic strategy for neurodegenerative diseases including PD through anti-inflammatory and antioxidant effects.

We previously synthesized and reported vinyl sulfone scaffolds as an Nrf2 activator. ${ }^{23-25}$ The lead compound (1) with the vinyl sulfone scaffold induced expression of Nrf2dependent antioxidant enzyme and relieved the production of inflammatory mediators through $\mathrm{Nrf2}$ signaling. ${ }^{232}$ Compound 1 also showed promising efficacy in the 1-methyl-4phenyl-1,2,3,6,-tetrahydropyridine (MPTP)-induced PD mouse model.

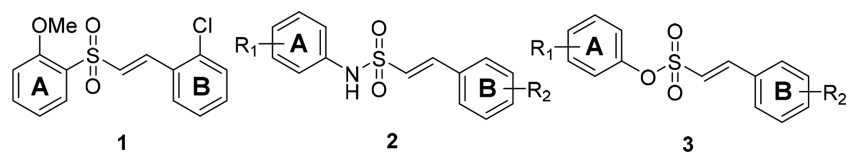

In this study, we replaced the sulfone moiety of compound 1 with a sulfonamide or sulfonate. Vinyl sulfonamides (2) and vinyl sulfonates (3) can be conveniently synthesized and also have an $\alpha, \beta$-unsaturated sulfone group, which is a highly activated Michael acceptor and may be responsible for activating Nrf2. Here, we report the development, synthesis, and biological evaluation of a series of vinyl sulfonamides and vinyl sulfonates. We observed that vinyl sulfonate scaffold enhanced Nrf2 activating efficacy. The most potent compound (3c) was evaluated for the expression of antioxidant enzymes and attenuated the production of inflammatory cytokines in BV-2 microglial cells and SH-SY5Y human neuroblastoma cells. Finally, the compound was tested for in vivo therapeutic effects on parkinsonism in the MPTP-induced mouse model.

\section{RESULTS AND DISCUSSION}

We further optimized previously developed compound $\mathbf{1}$ by modifying the $\alpha, \beta$-unsaturated sulfone position according to two categories: vinyl sulfonamides (2) or vinyl sulfonates (3). The synthetic pathway of vinyl sulfonamides (2) and vinyl sulfonates (3) are described in Scheme 1. Various styrenes $(4 \mathbf{a}-4 d)$ were prepared by the addition of sulfuryl chloride $\left(\mathrm{SO}_{2} \mathrm{Cl}_{2}\right)$ in dimethylformamide (DMF) for $3 \mathrm{~h}$ at $90{ }^{\circ} \mathrm{C}$ to produce (E)-2-phenylethene-1-sulfonyl chlorides (5a-5d). Condensation of $\mathbf{5 a - 5 d}$ with various anilines or phenols in the presence of triethylamine (TEA) in dichloromethane $\left(\mathrm{CH}_{2} \mathrm{Cl}_{2}\right)$ for $5 \mathrm{~h}$ at room temperature provided the sulfonamides $\mathbf{2 a}-\mathbf{2 e}$ and the sulfonates $\mathbf{3 a}-\mathbf{3 k}$, respectively.

The synthesized compounds were evaluated for $\mathrm{Nrf} 2$ activation in the optimized cell-based system. As we previously reported, this assay system assesses the ability of synthesized compounds to free $\mathrm{Nrf} 2$ from Keap1 and induce Nrf2 translocation to the nucleus using a genetically engineered stable cell-line. ${ }^{25,26}$ The efficacy of Nrf2 activation is described in Table 1 as $\mathrm{EC}_{50}$ values. In this assay, the previously developed compound 1 was confirmed to increase $\mathrm{Nrf} 2$ activation $\left(1, \mathrm{EC}_{50}=0.530 \mu \mathrm{M}\right)$.

First, we replaced the sulfone moiety with sulfonamide and observed that Nrf2 activation was minimal. In our previous study, we found that the addition of the methoxy (OMe) and chloride $(\mathrm{Cl})$ functional groups improved $\mathrm{Nrf} 2$ activation. We systematically attached a methoxy group to the 2-, 3-, and 4positions of the A ring and a chloride group to the $2^{\prime}-$-, $3^{\prime}$-, and $4^{\prime}$-positions of the $B$ ring. We found that addition of a methoxy
Scheme 1. Synthesis of Compounds 2 and $3^{a}$

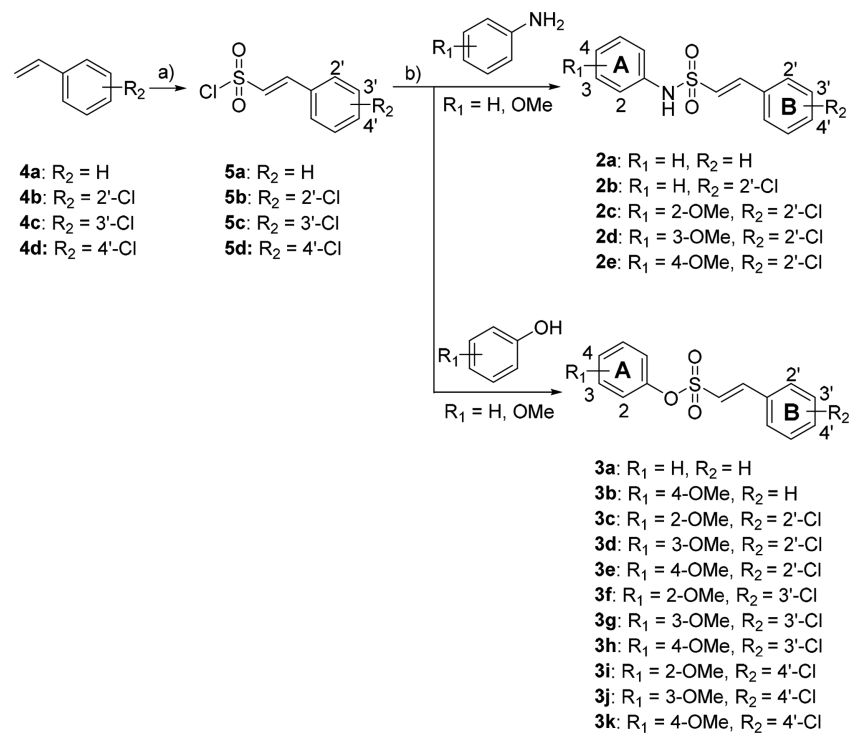

${ }^{a}$ Reagents and conditions: (a) $\mathrm{SO}_{2} \mathrm{Cl}_{2}$, DMF, $0{ }^{\circ} \mathrm{C}$ to r.t., $3 \mathrm{~h}$; (b) $\mathrm{Et}_{3} \mathrm{~N}, \mathrm{CH}_{2} \mathrm{Cl}_{2}$, r.t., 5 h.

Table 1. EC 50 Values against Nrf2 Activation of Synthesized Compounds

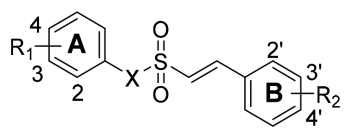

\begin{tabular}{|c|c|c|c|c|}
\hline compd. & $\mathrm{X}$ & $\mathrm{R}_{1}$ & $\mathrm{R}_{2}$ & Nrf2 $\mathrm{EC}_{50}(\mu \mathrm{M})^{a}$ \\
\hline $2 a$ & $\mathrm{~N}$ & $-\mathrm{H}$ & $-\mathrm{H}$ & $>10$ \\
\hline $2 b$ & $\mathrm{~N}$ & $-\mathrm{H}$ & $2^{\prime}-\mathrm{Cl}$ & $>10$ \\
\hline $2 c$ & $\mathrm{~N}$ & 2-OMe & $2^{\prime}-\mathrm{Cl}$ & $6.350 \pm 0.039$ \\
\hline $2 d$ & $\mathrm{~N}$ & 3-OMe & $2^{\prime}-\mathrm{Cl}$ & $>10$ \\
\hline $2 e$ & $\mathrm{~N}$ & 4-OMe & $2^{\prime}-\mathrm{Cl}$ & $>10$ \\
\hline $3 a$ & $\mathrm{O}$ & $-\mathrm{H}$ & $-\mathrm{H}$ & $1.040 \pm 0.015$ \\
\hline $3 b$ & $\mathrm{O}$ & 4-OMe & $-\mathrm{H}$ & $1.480 \pm 0.047$ \\
\hline $3 c$ & $\mathrm{O}$ & 2-OMe & $2^{\prime}-\mathrm{Cl}$ & $0.076 \pm 0.009$ \\
\hline $3 d$ & $\mathrm{O}$ & 3-OMe & $2^{\prime}-\mathrm{Cl}$ & $0.165 \pm 0.026$ \\
\hline $3 e$ & $\mathrm{O}$ & 4-OMe & $2^{\prime}-\mathrm{Cl}$ & $0.237 \pm 0.015$ \\
\hline $3 f$ & $\mathrm{O}$ & 2-OMe & $3^{\prime}-\mathrm{Cl}$ & $0.698 \pm 0.016$ \\
\hline $3 g$ & $\mathrm{O}$ & 3-OMe & $3^{\prime}-\mathrm{Cl}$ & $0.943 \pm 0.050$ \\
\hline $3 h$ & $\mathrm{O}$ & 4-OMe & $3^{\prime}-\mathrm{Cl}$ & $0.948 \pm 0.028$ \\
\hline $3 \mathbf{i}$ & $\mathrm{O}$ & 2-OMe & $4^{\prime}-\mathrm{Cl}$ & $1.334 \pm 0.024$ \\
\hline $3 \mathbf{j}$ & $\mathrm{O}$ & 3-OMe & $4^{\prime}-\mathrm{Cl}$ & $1.778 \pm 0.030$ \\
\hline $3 k$ & $\mathrm{O}$ & 4-OMe & $4^{\prime}-\mathrm{Cl}$ & $1.155 \pm 0.036$ \\
\hline $\mathbf{1}^{b}$ & & 2-OMe & $2^{\prime}-\mathrm{Cl}$ & $0.530 \pm 0.025$ \\
\hline $\mathbf{S F N}^{c}$ & & & & $0.580 \pm 0.024$ \\
\hline
\end{tabular}

${ }^{a}$ The Nrf2 functional assay was accomplished by a PathHunter U2OS Nrf2 nuclear translocation cell line (93-0821C3, DiscoveRx). U2OS cells were plated at 13,000 cells/well in triplicate with various compound concentrations for $6 \mathrm{~h}$. Nrf2 translocation, which is activation-dependent, was determined using a cell-based functional assay of the mean \pm standard error half maximal effective concentration $\left(\mathrm{EC}_{50}\right)$ values. ${ }^{b}$ Compound 1 developed as an $\mathrm{Nrf} 2$ activator in a previous study. ${ }^{24} \mathrm{SFN}$, sulforaphane: a well-known potent Nrf2 activator.

group to the 2-position of the A ring and a chloride to the 2 position of the $\mathrm{B}$ ring enhanced the $\mathrm{Nrf} 2$ activation relative to compound $2 \mathrm{a}\left(2 \mathrm{c} \mathrm{EC} \mathrm{EC}_{50}=6.35 \mu \mathrm{M}\right.$ vs $\left.2 \mathrm{a} \mathrm{EC}_{50}>10 \mu \mathrm{M}\right)$. Next, we substituted the sulfone moiety with sulfonate. Impressively, 
compound 3a showed Nrf2 activation in comparison to that of compound 2a $\left(3 \mathrm{a} \mathrm{EC}_{50}=1.04 \mu \mathrm{M}\right)$. We again systematically introduced the methoxy and chloride functional groups and observed Nrf2 activation. Similar to the results of a previous study, the vinyl sulfonates exhibited the most potent $\mathrm{Nrf} 2$ activation when 2 -OMe $(2-\mathrm{OMe}>3-\mathrm{OMe}>4-\mathrm{OMe})$ was added to $\mathrm{R}_{1}$ of the $\mathrm{A}$ ring and $2^{\prime}-\mathrm{Cl}\left(2^{\prime}-\mathrm{Cl}>3^{\prime}-\mathrm{Cl}>4^{\prime}-\mathrm{Cl}\right)$ was inserted into $R_{2}$ of the $B$ ring. The compounds $3 c-3 e$ with $2^{\prime}$ $\mathrm{Cl}$ on $\mathrm{B}$ ring exhibited potent $\mathrm{Nrf} 2$ activation compared to compound 1 . In these 11 series of compounds, $3 \mathrm{c}$ with $2-\mathrm{OMe}$ on A ring showed about 7-fold increase in Nrf2 activation than compound $1\left(3 \mathrm{c}, \mathrm{EC}_{50}=0.076 \mu \mathrm{M}\right.$, vs $\left.1, \mathrm{EC}_{50}=0.530 \mu \mathrm{M}\right)$. Thus, we selected $3 \mathrm{c}$ for further biological studies because it exhibited the most potent effect on $\mathrm{Nrf} 2$ activation $\left(\mathrm{EC}_{50}=\right.$ $0.076 \mu \mathrm{M}$; Figure 1a) and favorable metabolic stabilities (Table S1 in the Supporting Information).
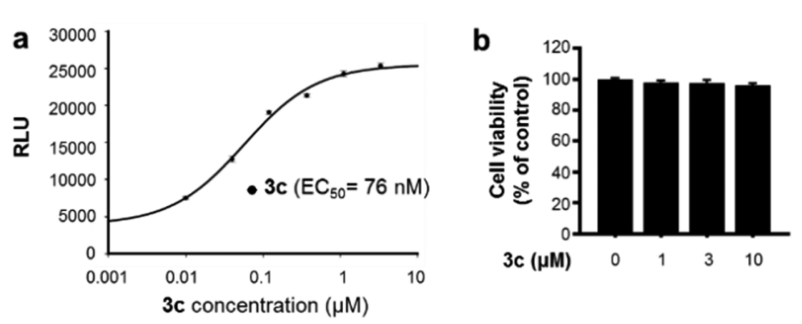

c
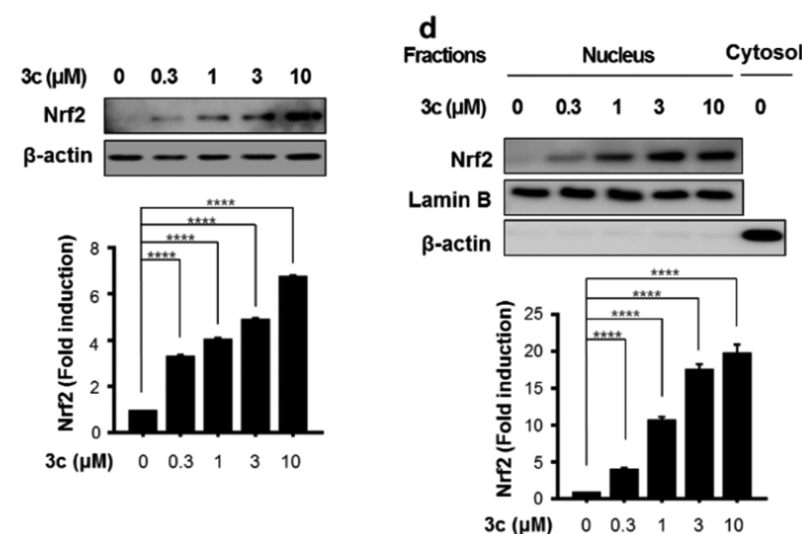

Figure 1. Nrf2 translocation to the nucleus is activated by 3c. (a) Nrf2 translocation assay of engineered U2OS cells was treated with various concentrations of 3c. (b) Viability assay of BV-2 cells treated with $3 \mathrm{c}$ for $6 \mathrm{~h}$. (c) After treatment with 3c $(0.3-10 \mu \mathrm{M})$, Nrf2 levels were analyzed by Western blotting using whole BV-2 microglial cells (d) and nuclear extracts. All experiments were performed at least in duplicate cultures, and the data were averaged and expressed as fold induction of untreated control \pm SEM. $* * * * P<0.001$ vs untreated control (paired $t$ test).

The cytotoxic potential of $3 \mathrm{c}$ was assessed, and nearly $100 \%$ cell survival was observed up to a concentration of $10 \mu \mathrm{M}$ $(98.0 \pm 1.2 \%$ at $1 \mu \mathrm{M}, 97.6 \pm 2.4 \%$ at $3 \mu \mathrm{M}, 95.9 \pm 1.6 \%$ at 10 $\mu \mathrm{M}$; Figure $1 \mathrm{~b})$. We then examined the effects on $\mathrm{Nrf} 2$ activation and expression of antioxidant enzymes in vitro. The results indicated that $3 \mathrm{c}$ increased $\mathrm{Nrf2}$ activation in a concentration-dependent manner. Figure 1c shows that treatment with $3 \mathrm{c}$ significantly increased nuclear $\mathrm{Nrf} 2$ protein levels in a concentration-dependent manner $(4.0 \pm 0.01$-fold at $0.3 \mu \mathrm{M}, 17.6 \pm 0.04$-fold at $10 \mu \mathrm{M})$. We also confirmed that total Nrf2 protein levels increased in a concentrationdependent manner $(3.3 \pm 0.02$-fold at $0.3 \mu \mathrm{M}, 6.7 \pm 0.03$ fold at $10 \mu \mathrm{M}$; Figure 1d).
We investigated whether the expression of ARE-targeted antioxidant enzymes was induced by $\mathrm{Nrf} 2$ activation. The enzyme HO-1 degrades heme to carbon monoxide, iron ions, and biliverdin. HO-1 activity ultimately has important physiological functions associated with cell protection. ${ }^{27,28}$ GCL, an antioxidant enzyme in the biosynthetic pathway for major cells, consists of modulatory (GCLM) and catalytic (GCLC) subunits. ${ }^{29}$ Real-time quantitative polymerase chain reaction (RT-qPCR) analysis revealed that the mRNA levels of HO-1, GCLM, and GCLC were upregulated by treatment with 3c (10.7-, 5.3-, and 2.3-fold, respectively; Figure 2a). Western blot analysis indicated that HO-1 and GCLM protein levels were significantly increased by 10.3-fold and 5.6-fold following treatment with $3 c$, respectively (Figure $2 b$ ).

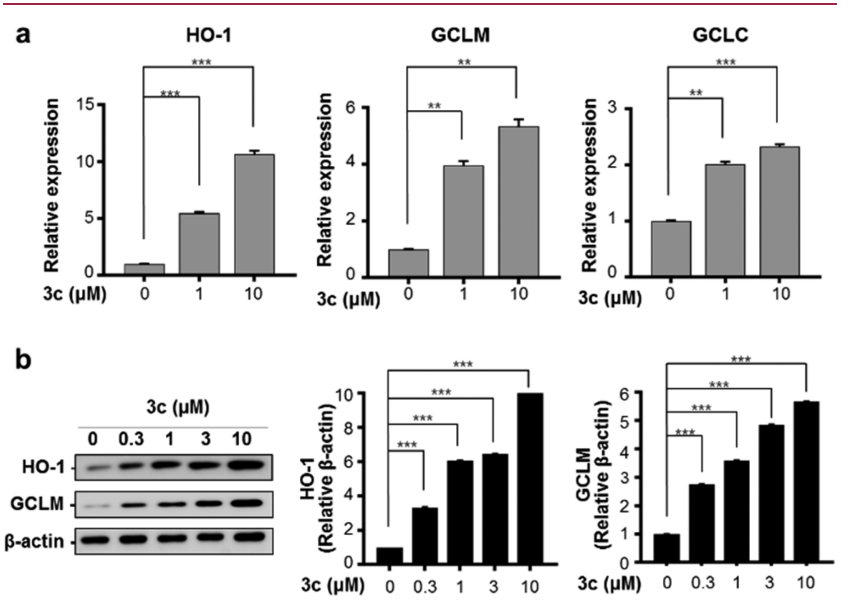

Figure 2. Compound $3 \mathrm{c}$ induces gene expression of $\mathrm{Nrf2}$-dependent antioxidant enzymes in BV-2 cells. (a) BV-2 cells were exposed to various concentrations of $3 \mathrm{c}$ for $3 \mathrm{~h}$ followed by RT-qPCR analysis of HO-1, GCLM, and GCLC expression. (b) BV-2 cells were treated with $3 \mathrm{c}$ for $12 \mathrm{~h}$, and protein levels of HO-1, GCLM, and GCLC were measured via Western blot analysis. The expression levels of antioxidant enzymes were normalized to $\beta$-actin. All experiments were performed at least in duplicate cultures, and the data were averaged and expressed as fold induction of untreated control \pm SEM. ** $P<0.01$, *** $P<0.005$ (paired $t$ test).

We also confirmed the antioxidant effects of 3c in SH-SY5Y cells. SH-SY5Y cells have been extensively used to investigate DAergic cell death induced by oxidative damage in PD studies. $^{30}$

NQO1 is a crucial factor in the metabolism and toxicity of quinone and acts as a detoxification agent in mitochondrial phase 2 metabolism. Western blot analysis revealed that the protein level of NQO1 increased 4.7-fold by treating with $3 \mathrm{c}$ (Figure 3). As observed in BV-2 cells, GCL protein levels were also concentration-dependently increased by treatment with $3 \mathrm{c}$ in SH-SY5Y cells. Both GCLM and GCLC protein levels were significantly upregulated by treatment with $3 c$, reaching 2.9and 1.5 -fold induction, respectively. These results indicate that $3 c$ quantitatively up-regulates the Nrf2/ARE pathway in BV-2 cells and SH-SY5Y.

We investigated whether $3 c$ relieves lipopolysaccharide (LPS)-induced inflammation by the down-regulation of nitric oxide (NO) synthase, iNOS, COX-2, IL- $1 \beta$, and TNF- $\alpha$ in microglial cells. To induce an inflammatory response, BV-2 cells were stimulated with LPS. $^{30}$ As shown in Figure 4, 3c significantly attenuated LPS-induced inflammatory enzymes and cytokines in BV-2 cells. Dramatic up-regulation of iNOS 

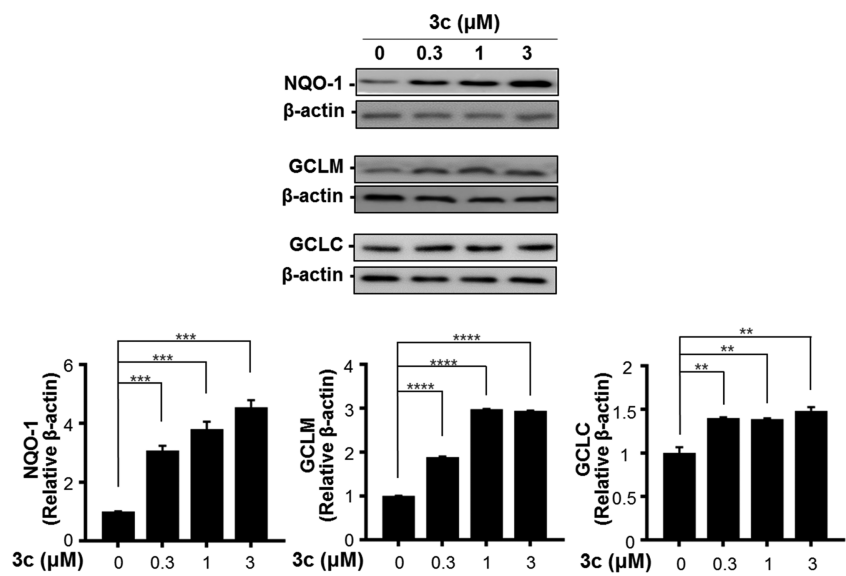

Figure 3. Compound $3 \mathrm{c}$ affects gene expression of Nrf2-dependent antioxidant enzymes in SH-SY5Y cells. SH-SY5Y cells were treated with $3 \mathrm{c}$ for 6 or $24 \mathrm{~h}$ and harvested for Western blot analysis. NQO1, GCLM, and GCLC protein levels were presented as relative amounts using $\beta$-actin as a loading control. All experiments were performed at least in duplicate cultures, and the data were averaged and expressed as fold induction of untreated control \pm SEM. $* * P<0.01, * * * P<$ $0.005, * * * * P<0.001$ (paired $t$ test).

and COX-2 by LPS was suppressed by $3 c$ at protein levels (Figure $4 \mathrm{a}$ ). The upregulation of iNOS and IL- $1 \beta$ induced by LPS were also significantly reduced by $3 \mathrm{c}$ at mRNA levels (Figure 4b). NO, which plans an important role in the pathogenesis of inflammation, was used as an inflammatory marker. ${ }^{31}$ We found the increase in NO production induced by LPS was significantly diminished by treatment with $3 \mathrm{c}$ by ELISA (Figure 4c). In addition, LPS-induced elevation of IL-6 and TNF- $\alpha$ were attenuated by $3 c$ in a concentrationdependent manner (Figure $4 d$ ).
We investigated whether $3 \mathrm{c}$ improves motor deficits using the MPTP-induced mouse model for PD. We evaluated the ability of mice to restore their motor ability by treatment with 3 c $(20 \mathrm{mg} / \mathrm{kg}$, p.o. $)$ for 3 consecutive days prior and subsequent to MPTP administration (four injections of MPTP, $2 \mathrm{~h}$ intervals; $20 \mathrm{mg} / \mathrm{kg}$, i.p. injection, Figure 5a).

PD-related motor dysfunction was tested using a coathanger test that included a vertical grid test ${ }^{32}$ established by our group. In behavioral tests, motor deficits in the MPTPinduced PD model were prevented by treatment with $3 \mathrm{c}$. Specifically, the turning time, descent time, and total time in MPTP-treated mice were restored to control levels by $3 \mathrm{c}$ (Figure 5b).

Similarly, coat-hanger tests also exhibited that 3c-treated mice improved abnormal movement compared to MPTPtreated mice and showed similar scores to the vehicle control (Figure 5c). MPTP-treated mice remained in their bases or moved horizontally (score: $1.9 \pm 0.1$ ), whereas mice treated with $3 c$ (score: $3.0 \pm 0.2$ ) showed a similar score to the vehicle control (score: $3.2 \pm 0.2$ ). Therefore, all data demonstrate that $3 c$ is highly effective in preventing PD-related movement disorders in MPTP-treated mouse models of PD (detailed information for statistical analysis is shown in the Supporting Information, Table S2).

We found that $3 c$ demonstrated antioxidant and antiinflammatory effects in vitro, so we then tested the neuroprotective effects on DAergic neurons in vivo using the MPTPinduced PD mouse model. The number of tyrosine hydroxylase ( $\mathrm{TH})$-negative neurons was reduced in MPTPtreated mice (up to $39 \pm 8.3 \%$ of the treated vehicle), but not in 3c-co-treated mice ( $P>0.001$ vs vehicle treatment). While the TH-positive cell density in striatum and SN was reduced after MPTP injection compared to controls, the cell density was significantly restored in 3c-treated mice (Figure 6a,b).

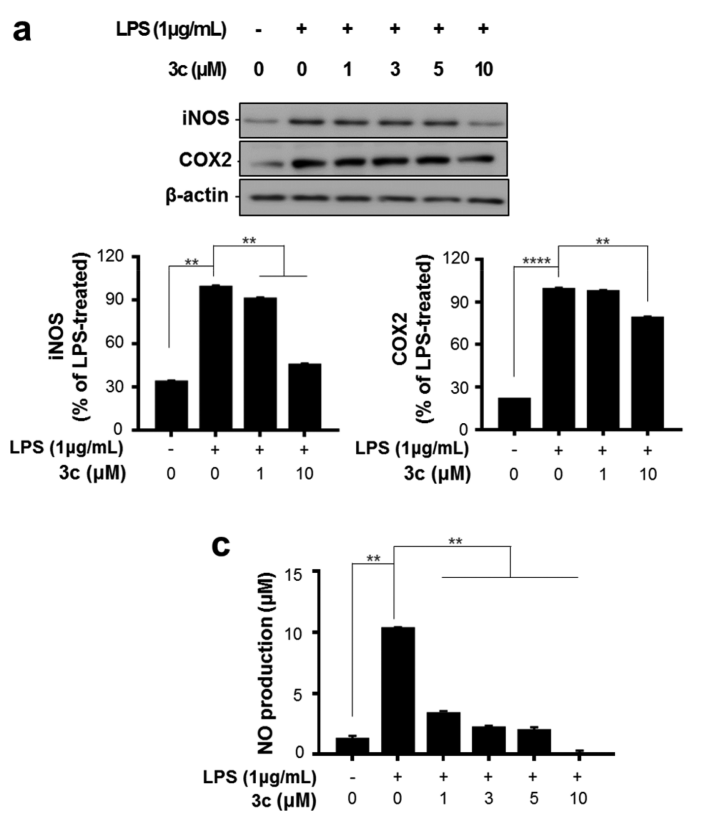

b
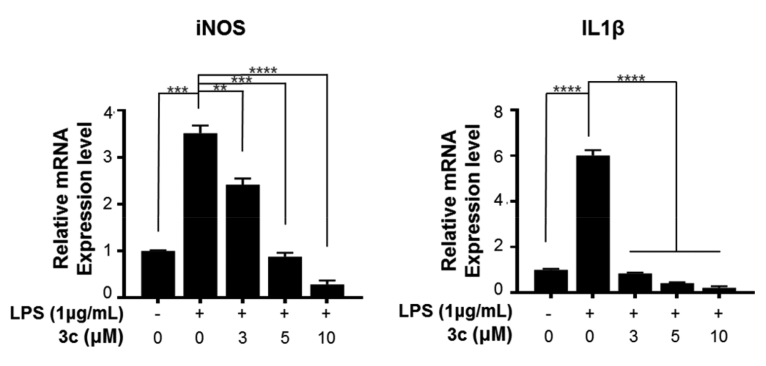

d
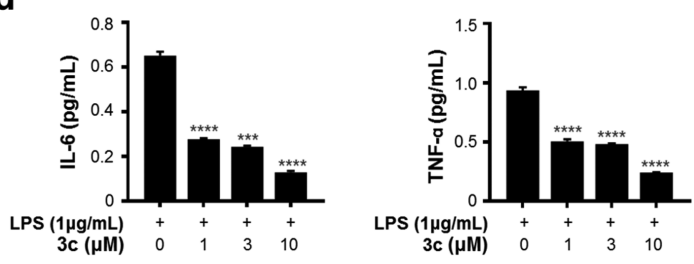

Figure 4. Expression levels of inflammation-related enzymes are decreased by $3 c$ in the concentration-dependent manner. (a-b) BV-2 cells were treated with $3 \mathrm{c}$ for $6 \mathrm{~h}$ and subsequently exposed to $1 \mu \mathrm{g} / \mathrm{mL}$ LPS for $12 \mathrm{~h}$. Protein and mRNA levels were quantitated and presented as relative levels. Data are presented as mean \pm SD $(n=3)$. (c-d) BV-2 cells were treated with $3 \mathrm{c}$ and exposed to $1 \mu \mathrm{g} / \mathrm{mL}$ LPS for $24 \mathrm{~h}$. All experiments were performed at least in duplicate cultures, and the data were averaged and expressed as relative values compared with the LPS-stimulated control \pm SEM. $* * P<0.01, * * * P<0.005, * * * * P<0.001$ vs LPS-stimulated control (paired $t$ test). 
a
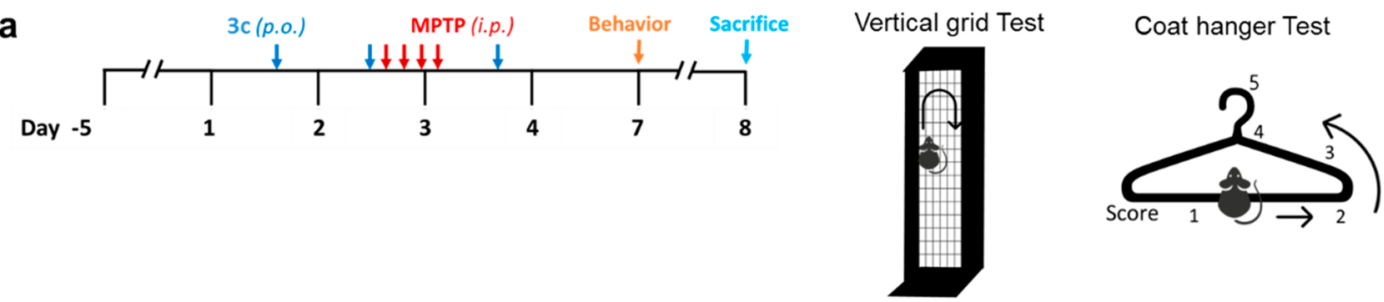

b

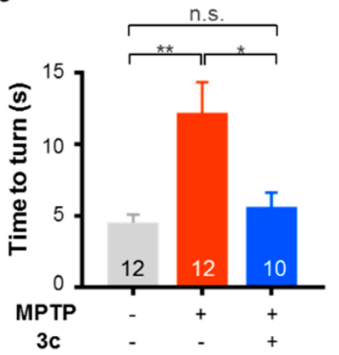

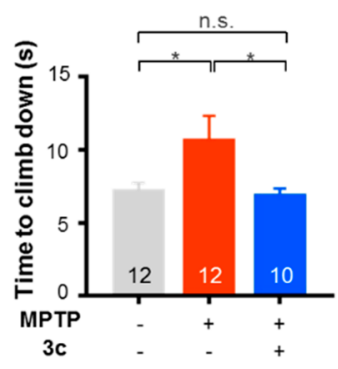

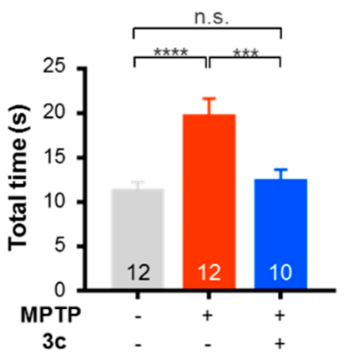

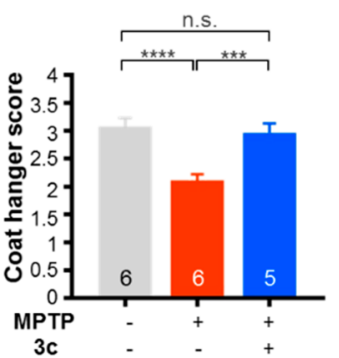

Figure 5. Compound 3c attenuates motor dysfunction in MPTP-treated mice. (a) Experimental schedule and behavior test diagram. Mice were administered $3 c(20 \mathrm{mg} / \mathrm{kg})$ or vehicle and treated with MPTP $(20 \mathrm{mg} / \mathrm{kg})$ four times after $24 \mathrm{~h} .(\mathrm{b}-\mathrm{c})$ Behavioral deficit recovery of the mice was evaluated by the vertical grid and coat-hanger tests. Results are shown as mean \pm SEM $(n=10-12$ per group). $* P<0.05$, $* * P<0.01, * * * P<$ $0.001, * * * * P<0.0001$, vs the MPTP-treated group (one-way ANOVA with Tukey's multiple comparisons test).

Compound $3 \mathrm{c}$ protected DAergic neurons in the striatum of the PD mouse model.
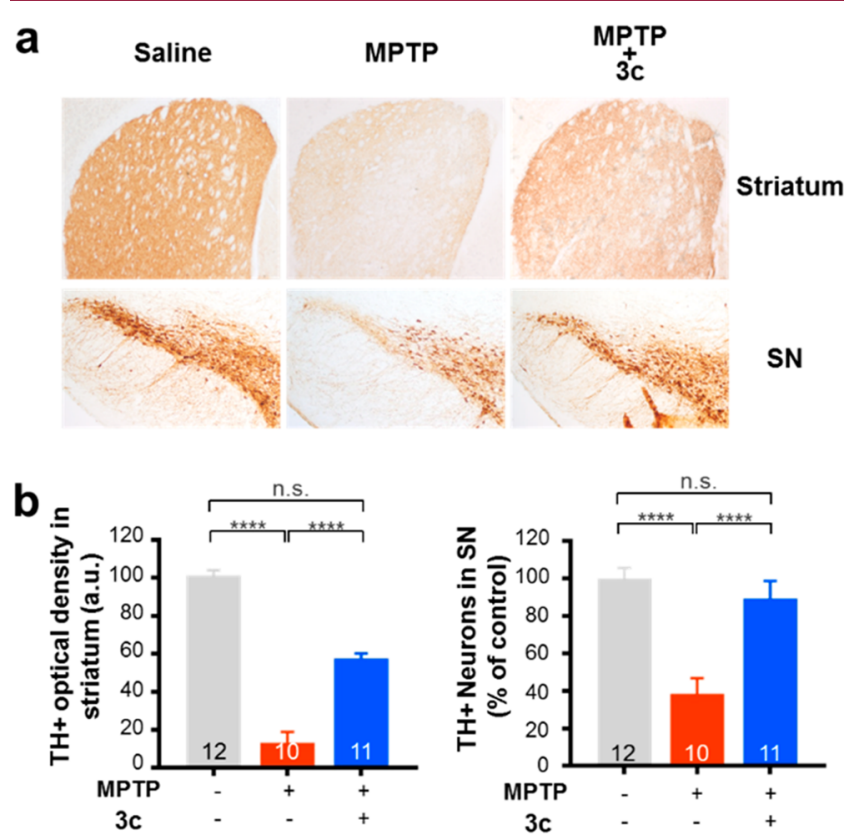

Figure 6. Compound $3 c$ protects DAnergic neurons in the MPTPinduced mouse model. (a) TH protein staining was performed using striatal and $\mathrm{SN}$ tissue sections of mice treated with vehicle or $3 \mathrm{c}$ (collected 7 days later). (b) Density of TH-positive neurons in the striatum and the number of $\mathrm{TH}$-positive neurons in the $\mathrm{SN}$ were analyzed. Both results are shown as mean \pm SEM. $* * * * P<0.0001$, vs the MPTP-treated group (one-way ANOVA with Tukey's multiple comparisons test).

We used the MPTP-induced PD model to determine whether $3 \mathrm{c}$ can suppress neuroinflammation in vivo. An ionized calcium-binding adapter molecule 1 (Iba-1) as a microglial marker was observed in the DAergic degeneration area of the $\mathrm{SN}$. Immunofluorescence intensity of Iba-1 in MPTP-injected mice was 4-fold higher than that of saline-injected mice.
Nonetheless, MPTP-injected mice treated with 3c significantly decreased Iba-1 immunoreactivity. In addition, TH-positive DAergic neurons were abundantly detected only in the absence of activated microglia in the merged image (Figure 7a,b).

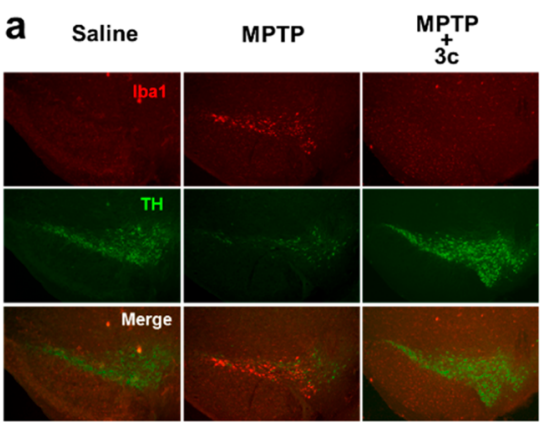

b

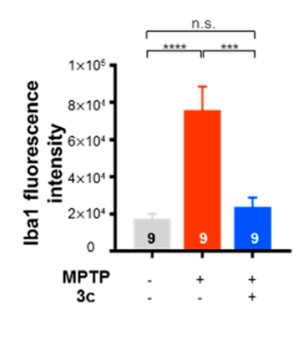

Figure 7. Compound $3 \mathrm{c}$ inhibited microglial activation in the MPTPinduced mouse model. Double immunofluorescence staining for $\mathrm{TH}$ (green) and Iba-1 (Red) in the brain 10 days after MPTP injection (i.p.). (a) TH and Iba-1 immunostaining was evident on the coronal section of SN. (b) Quantitative comparison of relative Iba-1 staining intensity between wild type $(n=9)$, 3c-treated $(n=9)$, and MPTPtreated $(n=9)$ mice at high magnification $(\times 4)$. Data are shown as mean \pm SEM. $* * * P<0.001, * * * * P<0.0001$, vs the MPTP-treated group (one-way ANOVA with Tukey's multiple comparisons test).

In conclusion, vinyl sulfonamide and vinyl sulfonate derivatives were developed and synthesized, and their effects on $\mathrm{Nrf} 2$ translocation and Nrf2 activation were biologically evaluated. Among them, the new vinyl sulfonate (3c) exhibited excellent $\mathrm{Nrf2}$ activation $\left(3 \mathrm{c}, \mathrm{EC}_{50}=76 \mathrm{nM}\right)$. We demonstrated that selected $3 \mathrm{c}$ induces the expression of Nrf2-dependent antioxidant enzymes and prevents the production of inflammatory mediators. In addition, 3c displayed potent in vivo therapeutic efficacy against motor dysfunction in MPTP-induced PD mice. The recovery of TH expression and anti-inflammatory responses in the $\mathrm{SN}$ and striatum were observed in this mouse model. In summary, the 
novel $\mathrm{Nrf} 2$ activator $3 \mathrm{c}$ has potential as an effective therapeutic agent for the treatment of PD.

\section{EXPERIMENTAL PROCEDURES}

Detailed synthetic procedures and analytical data for all compounds and experimental methods for biological studies are described in the Supporting Information.

\section{ASSOCIATED CONTENT}

\section{S Supporting Information}

The Supporting Information is available free of charge on the ACS Publications website at DOI: 10.1021/acsmedchemlett.9b00163.

Experimental methods for synthesis and biological studies; instrumental data for the synthesized compounds; ${ }^{1} \mathrm{H}$ - and ${ }^{13} \mathrm{C}-\mathrm{NMR}$ spectra of the final compounds (PDF)

\section{AUTHOR INFORMATION}

\section{Corresponding Author}

*E-mail: kdpark@kist.re.kr. Phone: +82-2-9585132. Fax: +822-9585189.

\section{ORCID}

Ki Duk Park: 0000-0002-7753-214X

\section{Author Contributions}

${ }^{\perp}$ J.W.C. and S.J.S. contributed equally to this work.

\section{Notes}

The authors declare no competing financial interest.

\section{ACKNOWLEDGMENTS}

This study was supported by a National Research Council of Science \& Technology grant by the South Korean government (MSIP, No. CRC-15-04-KIST) and the National Research Foundation of Korea (NRF-2018M3A9C8016849).

\section{ABBREVIATIONS}

ARE, antioxidant response element; DAergic, dopaminergic; GCLC, glutamate-cysteine ligase catalytic subunit; GCLM, glutamate-cysteine ligase regulatory subunit; HO-1, heme oxygenase; Iba-1, ionized calcium-binding adapter molecule 1; Keap1, Kelch-like ECH-related protein 1; LPS, lipopolysaccharide; MPTP, 1-methyl-4-phenyl-1,2,3,6-tetrahydropyridine; MS, multiple sclerosis; NO, nitric oxide; NQO-1, $\mathrm{NAD}(\mathrm{P}) \mathrm{H}$ :quinone oxidoreductase 1; Nrf2, nuclear factor E2related factor-2; PD, Parkinson's disease; SFN, sulforaphane; $\mathrm{SN}$, substantia nigra; $\mathrm{TH}$, tyrosine hydroxylase

\section{REFERENCES}

(1) Aarsland, D.; Creese, B.; Politis, M.; Chaudhuri, K. R.; Ffytche, D.-H.; Weintraub, D.; Ballard, C. Cognitive decline in Parkinson disease. Nat. Rev. Neurol. 2017, 13 (4), 217-231.

(2) Gratwicke, J.; Jahanshahi, M.; Foltynie, T. Parkinson's disease dementia: a neural networks perspective. Brain 2015, 138 (6), 145476.

(3) Branch, S. Y.; Chen, C.; Sharma, R.; Lechleiter, J. D.; Li, S.; Beckstead, M. J. Dopaminergic neurons exhibit an age-dependent decline in electrophysiological parameters in the mitopark mouse model of Parkinson's disease. J. Neurosci. 2016, 36 (14), 4026-37.

(4) Vogt Weisenhorn, D. M.; Giesert, F.; Wurst, W. Diversity matters - heterogeneity of dopaminergic neurons in the ventral mesencephalon and its relation to Parkinson's disease. J. Neurochem 2016, 139 (Suppl 1), 8-26.
(5) Asaithambi, A.; Ay, M.; Jin, H.; Gosh, A.; Anantharam, V.; Kanthasamy, A.; Kanthasamy, A. G. Protein kinase D1 (PKD1) phosphorylation promotes dopaminergic neuronal survival during 6OHDA-induced oxidative stress. PLoS One 2014, 9 (5), No. e96947.

(6) Dias, V.; Junn, E.; Mouradian, M. M. The role of oxidative stress in Parkinson's disease. J. Parkinsons Dis 2013, 3 (4), 461-91.

(7) Hou, Y.; Peng, S.; Li, X.; Yao, J.; Xu, J.; Fang, J. Honokiol alleviates oxidative stress-induced neurotoxicity via activation of $\mathrm{Nrf2}$. ACS Chem. Neurosci. 2018, 9, 3108-16.

(8) Sarkar, S.; Raymick, J.; Imam, S. Neuroprotective and therapeutic strategies against Parkinson's disease: recent perspectives. Int. J. Mol. Sci. 2016, 17 (6), 904.

(9) Amor, S.; Puentes, F.; Baker, D.; van der Valk, P. Inflammation in neurodegenerative diseases. Immunology 2010, 129 (2), 154-69.

(10) Bartels, A. L.; Leenders, K. L. Cyclooxygenase and neuroinflammation in Parkinson's disease neurodegeneration. Curr. Neuropharmacol 2010, 8 (1), 62-8.

(11) Lambert, T. W.; Soskolne, C. L.; Bergum, V.; Howell, J.; Dossetor, J. B. Ethical perspectives for public and environmental health: fostering autonomy and the right to know. Environ. Health Perspect. 2003, 111 (2), 133-7.

(12) Meredith, G. E.; Rademacher, D. J. MPTP mouse models of Parkinson's disease: an update. J. Parkinsons Dis 2011, 1 (1), 19-33.

(13) Tebay, L. E.; Robertson, H.; Durant, S. T.; Vitale, S. R.; Penning, T. M.; Dinkova-Kostova, A. T.; Hayes, J. D. Mechanisms of activation of the transcription factor Nrf2 by redox stressors, nutrient cues, and energy status and the pathways through which it attenuates degenerative disease. Free Radic Biol. Med. 2015, 88 (Pt B), 108-146.

(14) Kobayashi, E. H.; Suzuki, T.; Funayama, R.; Nagashima, T.; Hayashi, M.; Sekine, H.; Tanaka, N.; Moriguchi, T.; Motohashi, H.; Nakayama, K.; Yamamoto, M. Nrf2 suppresses macrophage inflammatory response by blocking proinflammatory cytokine transcription. Nat. Commun. 2016, 7, 11624.

(15) Baird, L.; Dinkova-Kostova, A. T. The cytoprotective role of the Keap1-Nrf2 pathway. Arch. Toxicol. 2011, 85 (4), 241-72.

(16) Magesh, S.; Chen, Y.; Hu, L. Small molecule modulators of Keap1-Nrf2-ARE pathway as potential preventive and therapeutic agents. Med. Res. Rev. 2012, 32 (4), 687-726.

(17) Done, A. J.; Traustadottir, T. Nrf2 mediates redox adaptations to exercise. Redox Biol. 2016, 10, 191-199.

(18) Suzuki, T.; Yamamoto, M. Stress-sensing mechanisms and the physiological roles of the Keap1-Nrf2 system during cellular stress. $J$. Biol. Chem. 2017, 292 (41), 16817-16824.

(19) Rojo, A. I.; Pajares, M.; Rada, P.; Nunez, A.; Nevado-Holgado, A. J.; Killik, R.; Van Leuven, F.; Ribe, E.; Lovestone, S.; Yamamoto, M.; Cuadrado, A. NRF2 deficiency replicates transcriptomic changes in Alzheimer's patients and worsens APP and TAU pathology. Redox Biol. 2017, 13, 444-451.

(20) Rojo, A. I.; Pajares, M.; Garcia-Yague, A. J.; Buendia, I.; Van Leuven, F.; Yamamoto, M.; Lopez, M. G.; Cuadrado, A. Deficiency in the transcription factor NRF2 worsens inflammatory parameters in a mouse model with combined tauopathy and amyloidopathy. Redox Biol. 2018, 18, 173-180.

(21) Burness, C. B.; Deeks, E. D. Dimethyl fumarate: a review of its use in patients with relapsing-remitting multiple sclerosis. CNS Drugs 2014, 28 (4), 373-87.

(22) Gold, R.; Kappos, L.; Arnold, D. L.; Bar-Or, A.; Giovannoni, G.; Selmaj, K.; Tornatore, C.; Sweetser, M. T.; Yang, M.; Sheikh, S. I.; Dawson, K. T. Placebo-controlled phase 3 study of oral BG-12 for relapsing multiple sclerosis. $N$ Engl J. Med. 2012, 367 (12), 1098-107.

(23) Lee, J. A.; Kim, J. H.; Woo, S. Y.; Son, H. J.; Han, S. H.; Jang, B. K.; Choi, J. W.; Kim, D. J.; Park, K. D.; Hwang, O. A novel compound VSC2 has anti-inflammatory and antioxidant properties in microglia and in Parkinson's disease animal model. Br. J. Pharmacol. 2015, 172 (4), 1087-1100.

(24) Woo, S. Y.; Kim, J. H.; Moon, M. K.; Han, S. H.; Yeon, S. K.; Choi, J. W.; Jang, B. K.; Song, H. J.; Kang, Y. G.; Kim, J. W.; Lee, J.; Kim, D. J.; Hwang, O.; Park, K. D. Discovery of vinyl sulfones as a 
novel class of neuroprotective agents toward Parkinson's disease therapy. J. Med. Chem. 2014, 57 (4), 1473-87.

(25) Choi, J. W.; Kim, S.; Park, J. H.; Kim, H. J.; Shin, S. J.; Kim, J. W.; Woo, S. Y.; Lee, C.; Han, S. M.; Lee, J.; Pae, A. N.; Han, G.; Park, K. D. Optimization of vinyl sulfone derivatives as potent nuclear factor erythroid 2-related factor 2 (Nrf2) activators for Parkinson's disease therapy. J. Med. Chem. 2019, 62, 811-830.

(26) Park, J. H.; Choi, J. W.; Ju, E. J.; Pae, A. N.; Park, K. D. Antioxidant and anti-Inflammatory activities of a natural compound, shizukahenriol, through Nrf2 activation. Molecules 2015, 20 (9), 15989-6003.

(27) Loboda, A.; Damulewicz, M.; Pyza, E.; Jozkowicz, A.; Dulak, J. Role of Nrf2/HO-1 system in development, oxidative stress response and diseases: an evolutionarily conserved mechanism. Cell. Mol. Life Sci. 2016, 73 (17), 3221-47.

(28) Li, H.; Wood, J. T.; Whitten, K. M.; Vadivel, S. K.; Seng, S.; Makriyannis, A.; Avraham, H. K. Inhibition of fatty acid amide hydrolase activates Nrf2 signalling and induces heme oxygenase 1 transcription in breast cancer cells. Br. J. Pharmacol. 2013, 170 (3), $489-505$.

(29) Takahashi, S.; Hisatsune, A.; Kurauchi, Y.; Seki, T.; Katsuki, H. Insulin-like growth factor 1 specifically up-regulates expression of modifier subunit of glutamate-cysteine ligase and enhances glutathione synthesis in SH-SY5Y cells. Eur. J. Pharmacol. 2016, 771, 99106.

(30) Kim, S. S.; Lim, J.; Bang, Y.; Gal, J.; Lee, S. U.; Cho, Y. C.; Yoon, G.; Kang, B. Y.; Cheon, S. H.; Choi, H. J. Licochalcone E activates Nrf2/antioxidant response element signaling pathway in both neuronal and microglial cells: therapeutic relevance to neurodegenerative disease. J. Nutr. Biochem. 2012, 23 (10), 1314-23. (31) Sharma, J. N.; Al-Omran, A.; Parvathy, S. S. Role of nitric oxide in inflammatory diseases. Inflammopharmacology 2007, 15 (6), 252259.

(32) Kim, S. T.; Son, H. J.; Choi, J. H.; Ji, I. J.; Hwang, O. Vertical grid test and modified horizontal grid test are sensitive methods for evaluating motor dysfunctions in the MPTP mouse model of Parkinson's disease. Brain Res. 2010, 1306, 176-83. 